\title{
Exploring market properties of policy-based reserve procurement for power systems
}

Ratha, Anubhav; Kazempour, Jalal; Virag, Ana; Pinson, Pierre

Published in:

Proceedings of 58th IEEE Conference on Decision and Control

Publication date:

2020

Document Version

Peer reviewed version

Link back to DTU Orbit

Citation (APA):

Ratha, A., Kazempour, J., Virag, A., \& Pinson, P. (2020). Exploring market properties of policy-based reserve procurement for power systems. In Proceedings of 58th IEEE Conference on Decision and Control (pp. 74987505). IEEE.

\section{General rights}

Copyright and moral rights for the publications made accessible in the public portal are retained by the authors and/or other copyright owners and it is a condition of accessing publications that users recognise and abide by the legal requirements associated with these rights.

- Users may download and print one copy of any publication from the public portal for the purpose of private study or research.

- You may not further distribute the material or use it for any profit-making activity or commercial gain

- You may freely distribute the URL identifying the publication in the public portal

If you believe that this document breaches copyright please contact us providing details, and we will remove access to the work immediately and investigate your claim. 


\title{
Exploring Market Properties of Policy-based Reserve Procurement for Power Systems
}

\author{
Anubhav Ratha, Jalal Kazempour, Ana Virag and Pierre Pinson
}

\begin{abstract}
This paper proposes a market mechanism for co-optimization of energy and reserve procurement in dayahead electricity markets with high shares of renewable energy. The single-stage chance-constrained day-ahead market clearing problem takes uncertain wind in-feed into account, resulting in optimal day-ahead dispatch schedule and an affine participation policy for generators for the real-time reserve provision. Under certain assumptions, the chance-constrained market clearing is reformulated as a convex quadratic program. Using tools from equilibrium modeling and variational inequalities, we explore the existence and uniqueness of a Nash equilibrium. Under the assumption of perfect competition in the market, we evaluate the satisfaction of desirable market properties, namely cost recovery, revenue adequacy, market efficiency, and incentive compatibility. To illustrate the effectiveness of the proposed market clearing, it is benchmarked against a deterministic cooptimization of energy and reserve procurement. Biased and unbiased out-of-sample simulation results for a power systems test case highlight that the proposed market clearing results in lower expected system operations cost than the deterministic benchmark, without the loss of any desirable market properties.
\end{abstract}

\section{INTRODUCTION}

Real-time balancing between supply and demand of electricity is challenging for the operation of power systems with high shares of intermittent renewable energy sources such as wind energy. Operational reserves for power systems are services traded in the market, in addition to energy, which ensure that deviations of actual wind power production from its day-ahead forecasts can be mitigated during real-time operation. Technical challenges aside, electricity markets also need to evolve such that cost-efficient market-based procurement and activation of reserves can be achieved.

Market-based procurement of reserves has been a topic of great interest in recent years, see [1], [2]. In current practice, illustrated in Figure 1a, the Market Operator (MO) estimates a system-wide parameter, Minimum Reserve Requirement (MRR) which is the minimum reserve capacity that must be procured to ensure a secure and efficient real-time balancing. Derived either from empirical studies or through probabilistic analysis of uncertainty [3], the MRR is then allocated among available flexible generators in a reserve capacity scheduling

The work of A. Ratha was supported by a Ph.D. grant provided by the Flemish Institute for Technological Research (VITO) and scholarship from Technical University of Denmark (DTU).

A. Ratha, J. Kazempour and P. Pinson are with the Department of Electrical Engineering, Technical University of Denmark, Lyngby, Denmark. \{arath, seykaz, ppin\}@elektro.dtu.dk

A. Ratha and A. Virag are with the Flemish Institute of Technological Research (VITO), Boeretang 200, 2400 Mol, Belgium and with EnergyVille, ThorPark 8310, 3600 Genk, Belgium. \{anubhav.ratha, ana.virag\}evito.be

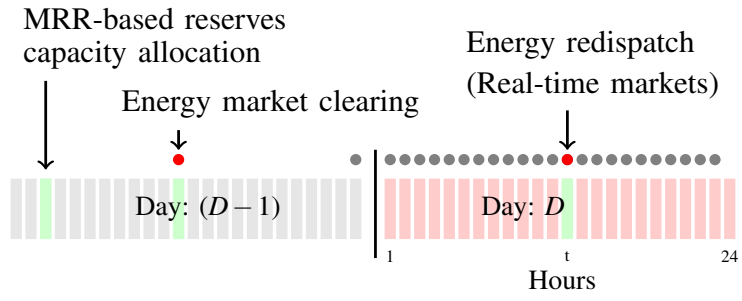

(a) Prevalent sequential reserve and energy procurement

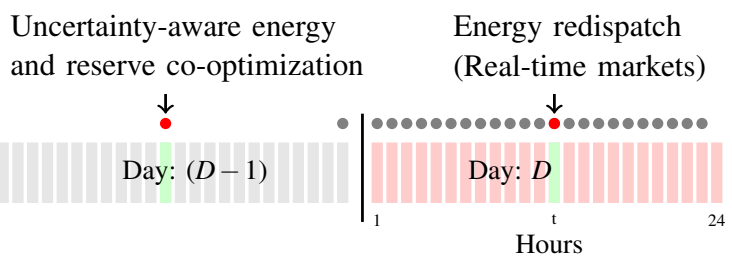

(b) State-of-the-art stochastic market clearing

Uncertainty-aware energy

and reserve co-optimization

+ Affine policy-based recourse

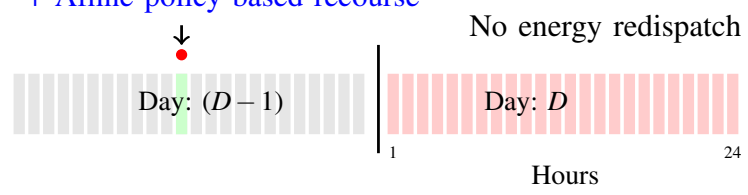

(c) Proposed chance-constrained policy-based reserves

Fig. 1: Comparison of market clearing mechanisms for energy and reserve. Each (•) represents a market clearing.

market prior to the day-ahead market clearing ${ }^{1}$. Finally, energy redispatch cost arises due to real-time markets that are necessary to activate the reserve energy from flexible generators to exactly meet the deviations. Faced with the situation to integrate an increasing amount of intermittent generation, over-estimation of MRR and thereby over-dimensioning of reserves has become a practice that increases the cost of operating power systems.

With the strong coupling that exists between the supply of energy and provision of reserves, recent studies have argued for co-optimization of energy and reserves in day-ahead markets [5], shown in Figure 1b. Primarily, two research streams have shaped this discussion. The first adopts scenario-based stochastic programming methods, for example in [6] and [7], to select reserves in the day-ahead stage such that the cost of activated reserves during real-time is optimal for the

\footnotetext{
${ }^{1}$ This practice is followed by several European electricity markets (except a few markets such as the Italian electricity market) [4]. Markets in the US follow a deterministic co-optimization to procure energy and reserves, similar to the benchmark discussed in Case Study (Section IV).
} 
expected realization of uncertainty and feasible for all the scenarios in the sample set considered. A very large number of scenarios is required to represent the uncertainty well and to ensure good out-of-sample performance, at the cost of higher computational need. The other research stream uses robust optimization techniques, such as in [8], [9] and [10], to allocate and operate reserves such that the cost of system operation is optimal with respect to the worst-case outcome and is feasible for any outcome of uncertainty within a parameterized uncertainty set. A market for reserves based on control policies was first proposed in [8], built upon a robust optimization model. While introducing computational ease, this approach by design results in a conservative solution and relies on meticulous study of uncertainty sets. Despite the new market direction introduced by [8], the market properties and pricing scheme were not elaborated upon.

In this paper, inspired by the control policy-based reserves discussed in [8], we propose a market clearing mechanism based on chance-constrained programming that co-optimizes energy and reserves at the day-ahead stage, as shown in Figure 1c. Chance-constrained programming, as highlighted in [11], provides a practical approach addressing drawbacks of the aforementioned techniques. Although it necessitates an additional step of convexification of chance constraints (see [12], [13]), it exhibits good out-of-sample performance and adjustable conservativeness at low computational cost. Based on the effectiveness of linear decision rules in decisionmaking under uncertainty, as examined in [14], we select an affine reserve policy such that participation in reserve activation during real-time operation stage is a linear function of the total balancing need. In addition to the optimal dimensioning of reserves, policy-based procurement of reserves has the advantage of resulting in efficient pricing for the reserve services while removing the requirement of realtime markets for reserve activation and associated energy redispatch cost. Under the assumption of perfect competition, aided by a study of Nash equilibria using tools from variational inequalities [15] in our proposed market clearing, we explore the existence and uniqueness of market equilibrium and the satisfaction of desirable market properties, namely cost recovery for agents, revenue adequacy for the MO, market efficiency and incentive compatibility [16].

We follow the analytical convexification of probabilistic chance constraints, introduced in [11], which results in a conic programming problem. Further, we use the direction suggested in [17] to simplify the conic constraints to linear formulations. In a case study built around a power system with a high share of installed wind power capacity and using biased and unbiased out-of-sample simulations, we benchmark the proposed market clearing method against a deterministic power and reserve co-optimization. This enables us to quantify the reduction in expected total system operations cost by the adoption of our proposed market clearing, under adverse realizations of uncertainty.

The paper is organized as follows: section II presents the proposed co-optimization of energy and reserves, stating the underlying assumptions and discussing its mathemati- cal interpretations as an equilibrium problem and a noncooperative game. Section III evaluates the satisfaction of market properties, while results of the numerical case study are presented and discussed in section IV. Finally, conclusions and future work are discussed in section $\mathrm{V}$.

\section{PROBLEM FORMULATION}

The following considers a single-node power system comprising of a set of flexible generators, $g \in \mathcal{G}$, a set of wind farms, $k \in \mathcal{K}$, and an inflexible aggregated load, $D$.

\section{A. Preliminaries}

It is assumed that perfect competition exists in the market, such that no market participant exhibits strategic behavior. The wind farms are assumed to have zero marginal cost of production and excess wind spillage is considered free. Further, uncertainty in the form of wind forecast errors is considered to be the only source of uncertainty in the system. To avoid non-convexities introduced by the commitment status of generators, it is assumed that only scheduled generators participate in the day-ahead market clearing. The value of lost load as well as the price caps for the day-ahead market prices is considered to be $€ 500 / \mathrm{MWh}$.

\section{B. Modeling of Uncertainty}

In the day-ahead market clearing stage, forecast errors in power production from the wind farm $k$ are modeled to follow a zero-mean Normal distribution, $\mathcal{N}\left(0, \sigma_{k}\right)$ centered around the best available point forecast, $\hat{W}_{k}$. The value of $\sigma_{k}$ should be estimated from historical forecast errors for each wind farm location and time of the day.

Assumption 1: The standard deviations of the zero-mean normally distributed random forecast errors of wind farms, $\sigma_{k}, \forall k \in \mathcal{K}$, are temporally and spatially uncorrelated with respect to hours of the day and among wind farm sites, respectively.

Remark 1: The assumption of spatial independence of forecast errors is realistic for markets operating over a large geographical area, as discussed in [11]. The absence of temporal correlation is an assumption adopted in this paper to allow for hourly decoupling of the problem.

Under Assumption 1, the total error or deviation during real-time is defined as

$$
\Delta=\sum_{k \in \mathcal{K}} \delta_{k}
$$

where $\delta_{k}$ refers to the error in forecast for the wind farm $k$. As a result, the uncertain parameter $\Delta$ is assumed to be drawn from a multivariate Normal distribution, $\mathcal{N}\left(\mathbf{0}_{N_{k}}, \Sigma\right)$, where $\Sigma=\operatorname{diag}\left(\sigma_{1}^{2}, \sigma_{2}^{2}, \ldots, \sigma_{N_{k}}^{2}\right)$.

\section{Chance-constrained Policy-based Reserves}

Considering $\alpha_{g}$ as a participation factor in the provision of reserves by generator $g$, we define an affine reserve policy such that when activated in real-time, the total generation from $g$ is given by $\tilde{p}_{g}=p_{g}-\Delta \alpha_{g}$.

In the proposed day-ahead market clearing mechanism, optimal hourly reserve policies characterized by $\alpha_{g}$, in 
addition to the nominal hourly energy dispatch, $p_{g}$ for each flexible generator are decided by the centralized MO. The reserve policies, which are then activated during realtime operation, define each generator's participation in the imbalance mitigation. Facing the uncertainty in wind power forecast errors $\Delta$, MO's objective is to minimize the expected cost of operating the power system. We consider quadratic costs of generation and simplify the probabilistic expectation term in the objective function, using the zero-mean property of forecast errors, as discussed in [11]. Further, we consider a single-node model of the transmission grid. The chanceconstrained optimization problem solved by the MO for joint clearing of energy and reserves is formulated as

$$
\begin{aligned}
& \min _{p_{g}, \alpha_{g}} \quad \sum_{g \in \mathcal{G}}\left[C_{g}^{Q}\left[p_{g}^{2}+\left(\mathbf{e}^{\top} \Sigma \mathbf{e}\right) \alpha_{g}^{2}\right]+C_{g}^{L} p_{g}\right] \\
& \text { s.t. } \quad \sum_{g \in \mathcal{G}} p_{g}+\sum_{k \in \mathcal{K}} \hat{W}_{k}=D \\
& \sum_{g \in \mathcal{G}} \alpha_{g}=1 \\
& \mathbb{P}\left[p_{g}-\Delta \alpha_{g} \geq 0\right] \geq\left(1-\varepsilon_{g}\right), \forall g \in \mathcal{G} \\
& \mathbb{P}\left[p_{g}-\Delta \alpha_{g} \leq p_{g}^{\max }\right] \geq\left(1-\varepsilon_{g}\right), \forall g \in \mathcal{G} \\
& \mathbb{P}\left[\Delta \alpha_{g} \leq R_{g}^{\mathrm{DN}, \max }\right] \geq\left(1-\varepsilon_{g}\right), \forall g \in \mathcal{G} \\
& \mathbb{P}\left[-\Delta \alpha_{g} \leq R_{g}^{\mathrm{UP}, \max }\right] \geq\left(1-\varepsilon_{g}\right), \forall g \in \mathcal{G} \\
& 0 \leq \alpha_{g} \leq 1, \forall g \in \mathcal{G}
\end{aligned}
$$

where $C_{g}^{Q}$ and $C_{g}^{L}$ are the quadratic and linear production costs of generator $g$ and $\mathbf{e} \in \mathbb{R}^{N_{k}}$ is a vector of all ones. Further, $p_{g}^{\max }, R_{g}^{\mathrm{DN}, \mathrm{max}}$ and $R_{g}^{\mathrm{UP} \text {, max }}$ are the maximum production capacity, maximum downward reserve capacity and maximum upward reserve capacity available with generator $g$, respectively. $\mathbb{P}[\cdot]$ denotes probability and $\varepsilon_{g}$ is the probability of the output from generator $g$ to exceed the maximum and minimum limits of production and reserve capacity.

Constraint (2b) ensures the supply-demand balance for available point forecasts of wind power production, whereas (2c) ensures that the total deviation from point forecasts is exactly mitigated in real-time. These two constraints are deterministic and independent of wind power production realizations, thus ensuring security of supply for the inflexible demand for all realizations of the uncertainty.

Chance constraints $(2 \mathrm{~d})-(2 \mathrm{~g})$ limit the probability of violation of bounds on net generation and activated reserves for all realizations of the uncertain parameter $\Delta$ to at most $\varepsilon_{g}$. The choice of risk parameter $\varepsilon_{g}$ influences the conservativeness of the market clearing solution, with smaller values leading to a higher cost of operation. To study properties of the market clearing mechanism (2), we now introduce a linearized reformulation of the chance constraints inspired by [18], by adopting the following assumption.

Assumption 2: The participation factors, $\alpha_{g}, \forall g \in \mathcal{G}$, characterizing the affine reserve policy are non-negative, as enforced by (2h).

Remark 2: While it is intuitive to assume that $\alpha_{g}$ should be considered non-negative, it may be beneficial to drop this assumption when working with a more realistic power

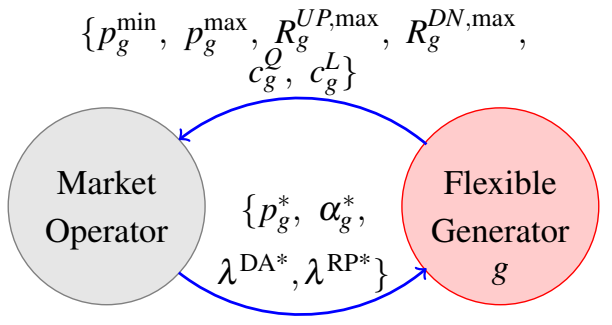

Fig. 2: Interaction between a flexible generator $g$ and the market operator. Superscript * denotes optimal values.

system model that considers the transmission network or has elements such as energy storage. In that case, the resulting problem is a second-order cone program, evaluating the market properties of which is left for future work.

Under Assumptions 1 and 2, using [19, Theorem 10.4.1], the problem (2) can be exactly reformulated as a convex quadratic program in $\left(p_{g}, \alpha_{g}\right)$, denoted by $\mathcal{P}_{\mathrm{cc}}$, i.e.,

$$
\begin{array}{ll}
\min _{p_{g}, \alpha_{g}} & \sum_{g \in \mathcal{G}}\left[C_{g}^{Q}\left[p_{g}^{2}+\left(\mathbf{e}^{\top} \Sigma \mathbf{e}\right) \alpha_{g}^{2}\right]+C_{g}^{L} p_{g}\right] \\
\text { s.t. } & \sum_{g \in \mathcal{G}} p_{g}+\sum_{k \in \mathcal{K}} \hat{W}_{k}=D: \quad \lambda^{\mathrm{DA}} \\
& \sum_{g \in \mathcal{G}} \alpha_{g}=1: \quad \lambda^{\mathrm{RP}} \\
& p_{g}-\Phi_{\left(1-\varepsilon_{g}\right)}^{-1} \alpha_{g} \sqrt{\left(\mathbf{e}^{\top} \Sigma \mathbf{e}\right)} \geq 0, \forall g \in \mathcal{G} \\
& p_{g}+\Phi_{\left(1-\varepsilon_{g}\right)}^{-1} \alpha_{g} \sqrt{\left(\mathbf{e}^{\top} \Sigma \mathbf{e}\right)} \leq p_{g}^{\max }, \forall g \in \mathcal{G} \\
& -\Phi_{\left(1-\varepsilon_{g}\right)}^{-1} \alpha_{g} \sqrt{\left(\mathbf{e}^{\top} \Sigma \mathbf{e}\right)} \leq R_{g}^{\mathrm{DN}, \max }, \forall g \in \mathcal{G} \\
& \Phi_{\left(1-\varepsilon_{g}\right)}^{-1} \alpha_{g} \sqrt{\left(\mathbf{e}^{\top} \Sigma \mathbf{e}\right)} \leq R_{g}^{\mathrm{UP}, \max }, \forall g \in \mathcal{G} \\
& 0 \leq \alpha_{g} \leq 1, \forall g \in \mathcal{G},
\end{array}
$$

where $\Phi_{(\cdot)}^{-1}$ is the inverse cumulative distribution function or the quantile function of the standard Normal distribution $\mathcal{N}(0,1)$. The risk parameter $\varepsilon_{g}$ is chosen from the domain $[0,0.5)$, such that $\Phi_{\left(1-\varepsilon_{g}\right)}^{-1}$ is always positive [18]. Note that all constraints are linear.

In problem $\mathcal{P}_{\mathrm{cc}}$, the dual variables of constraints ( $\left.3 b\right)$ and (3c), provide the market clearing price for energy, $\lambda^{\mathrm{DA}}$ and reserve policies, $\lambda^{\mathrm{RP}}$, which are then used for remuneration of the generators for energy $p_{g}$ and reserve participation $\alpha_{g}$, respectively. The interaction between a flexible generator, $g$ and the market operator is illustrated in Figure 2.

In practice, it can be expected that hour-ahead of realtime operation, when the uncertainty in forecast is considerably reduced compared to the day-ahead stage, the market operator broadcasts the best estimate of "realized forecast errors" $\Delta$ which is used by flexible generators with non-zero $\alpha_{g}$ to adjust their real-time production for the next hour. Any remaining instantaneous errors are considered to be handled by primary frequency control or spinning reserves. This eliminates the need for an additional real-time balancing market, prevalent in current practice, in the proposed policybased market clearing mechanism. 


\section{Mathematical Interpretations of Problem $\mathcal{P}_{c c}$}

1) As an equilibrium problem: In alignment with the discussions in [20] and [21], problem $\mathcal{P}_{\text {cc }}$ can be expressed as an equilibrium problem, wherein each market player solves a profit maximization problem while being connected through market clearing conditions (supply-demand balance). For the flexible generators, the profit maximization problem is

$$
\forall g \in \mathcal{G}\left\{\begin{aligned}
\max _{p_{g}, \alpha_{g}} & \left(\lambda^{\mathrm{DA}} p_{g}+\lambda^{\mathrm{RP}} \alpha_{g}\right) \\
& \quad-\left[C_{g}^{Q}\left(p_{g}^{2}+\left(\mathbf{e}^{\top} \Sigma \mathbf{e}\right) \alpha_{g}^{2}\right)+C_{g}^{L} p_{g}\right] \\
\text { s.t. } & (3 \mathrm{~d})-(3 \mathrm{~h}) .
\end{aligned}\right.
$$

Unlike the flexible generators, the wind farms have no profit maximization strategy considering they have a zero cost of production and no cost associated with spillage of excess wind. Similarly, inflexible demand is considered to have no profit maximization role in problem $\mathcal{P}_{\text {cc }}$. However, the MO enforces the constraints (3b) and (3c), which are the coupling constraints that connect the flexible generators, wind farms and the inflexible demand.

2) As a non-cooperative game: The optimization problem $\mathcal{P}_{\text {cc }}$ can be interpreted as a non-cooperative game among two sets of players. First, the flexible generators $g \in \mathcal{G}$, while operating within constraints of their production and reserve capacities, try to maximize their profits (or minimize their costs) from participation in the market for energy and reserves. Second, the MO who acts as a "price setter" to ensure that the market price for energy and reserves are set at as low values as possible, so that the inflexible demand $D$ can be met at the lowest cost possible.

We define $x_{g}=\left[\begin{array}{ll}p_{g} & \alpha_{g}\end{array}\right]^{\top}$ as the decision vector for the flexible generator $g$, such that $x_{g} \in K_{g}$ where $K_{g} \subset \mathbb{R}_{+}^{2}$ is its strategy set, from which its choices for the bid quantity $p_{g}$ and participation factor $\alpha_{g}$ are drawn. Similarly, we define $\Lambda=\left[\lambda^{\mathrm{DA}} \lambda^{\mathrm{RP}}\right]^{\top}$ as the decision vector for the MO, such that $\Lambda \in K_{M O}$ where $K_{M O} \subset \mathbb{R}_{+}^{2}$. The cost function for the flexible generator $g$ can thus be expressed as

$$
J_{g}\left(x_{g}, \Lambda\right)=\left(x_{g}^{\top} Q_{g} x_{g}+L_{g}^{\top} x_{g}\right)-\Lambda^{\top} x_{g}, \forall x_{g} \in K_{g},
$$

where $\Lambda$ denotes cleared market prices and represents decisions made by all other participants of the game i.e. the MO and flexible generators other than $g$, in the cost function for $g$. The costs $Q_{g}=\left[\begin{array}{cc}C_{g}^{Q} & 0 \\ 0 & C_{g}^{Q}\left(\mathbf{e}^{\top} \Sigma \mathbf{e}\right)\end{array}\right]$ and $L_{g}=\left[\begin{array}{c}C_{g}^{L} \\ 0\end{array}\right]$ represent the quadratic and linear costs for $g$.

Similarly, the MO is subject to a cost function which can be expressed as

$$
J_{M O}\left(\Lambda, x_{g}\right)=S^{\top} \Lambda, \forall \Lambda \in K_{M O},
$$

where $S=\left[\begin{array}{c}-\sum_{g \in \mathcal{G}} p_{g}-\sum_{k \in \mathcal{K}} \hat{W}_{k}+D \\ -\sum_{g \in \mathcal{G}} \alpha_{g}+1\end{array}\right]$ represents the decisions made by other players, i.e. all flexible generators $g \in \mathcal{G}$.

\section{EVALUATION OF MARKET PROPERTIES}

In this section, the problem definition and interpretations presented in section II are used to evaluate market properties of problem $\mathcal{P}_{\mathrm{cc}}$.

\section{A. Existence and Uniqueness of Nash equilibrium (NE)}

Let $\Gamma\left(\mathcal{Z}, K,\left\{J_{i}\right\}_{i \in Z}\right)$ denote the non-cooperative game among the flexible generators and the market operator, where $z=(\mathcal{G} \cup\{M O\})$ denotes the set of all players and $K=$ $\prod_{i} K_{i}=\left(K_{1} \times K_{2} \times \cdots \times K_{N_{g}} \times K_{M O}\right)$ denotes the strategy set for the game. Further, the decision variables of all players can be stacked to define a simultaneous strategy vector $z=\left[\begin{array}{llll}x_{1}^{\top} & \cdots & x_{N_{g}}^{\top} \Lambda^{\top}\end{array}\right]^{\top}$, such that $z \in \mathbb{R}^{2\left(N_{g}+1\right)}$ contains the strategy decisions of each of the players as a response to other players' actions.

Proposition 1: For the non-cooperative game among flexible generators and the market operator, $\Gamma\left(\mathcal{Z}, K,\left\{J_{i}\right\}_{i \in Z}\right)$, a Nash equilibrium exists.

Proof: A simultaneous strategy vector $z^{*} \in K$ is a Nash equilibrium if and only if

$$
J_{i}\left(z_{i}^{*}, z_{-i}^{*}\right) \leq J_{i}\left(z_{i}, z_{-i}^{*}\right), \forall z_{i} \in K_{i}, \forall i \in Z .
$$

To prove that Nash equilibria exist for the game $\Gamma$, we employ a theorem presented in [22, Theorem 1]. In the game $\Gamma$, each flexible generator $g$ has a strategy set $K_{g}$ formed by the upper and lower bounds of production and reserve capacities, while the market operator has a strategy set $K_{M O}$ which is formed by the non-negativity bounds as well as price caps set for the market clearing. This satisfies the condition of compactness and convexity for $K_{i}$. Further, the cost function $J_{g}$ in equation (5) is quadratic and thus, continuous over $z$ and convex over $x_{g}$ for fixed values of $x_{g^{\prime}}=\bar{x}_{g^{\prime}}, \forall g^{\prime} \in(\mathcal{G}-\{g\})$ and $\Lambda=\bar{\Lambda}$. Likewise, the market operator's cost function $J_{M O}$, given by equation (6) is linear and continuous in $z$ and convex over $\Lambda$ for fixed values of $x_{g}=\bar{x}_{g}, \forall g \in \mathcal{G}$. From [22, Theorem 1], this proves that at least one Nash equilibrium exists for the game $\Gamma$.

The vector(s) $z^{*}$ are a set of strategies for the $i$ players of the game $\Gamma$ in which each player chooses the best response to other players' decisions, implying that no player can lower their cost by unilaterally deviating their action from $z_{i}^{*}$ to any other feasible point, $\tilde{z}_{i}$.

Proposition 2: For the non-cooperative game among flexible generators and market operator, $\Gamma\left(Z, K,\left\{J_{i}\right\}_{i \in Z}\right)$ a unique Nash equilibrium exists.

Proof: This is proven by employing tools from Variational Inequalities (VIs) and their equivalence with Nash equilibria. For the game $\Gamma$, under the assumption that the game is played only by market players who have been dispatched or committed, the strategy sets $K_{i}$ for flexible generators are compact, convex and nonempty. Further, as discussed in Proposition 1, the cost functions $J_{i}\left(z_{i}, z_{-i}\right)$ for every fixed $z_{-i} \in K_{-i}$ are differentiable. Upon satisfaction of the above conditions, [15, Proposition 1.4.2] allows us to express the problem of finding Nash equilibria for $\Gamma$ as a $\mathrm{VI}(F, K)$ problem with

$$
F(z)=\left[\begin{array}{c}
\nabla_{1} J_{1}\left(z_{1}, z_{-1}\right) \\
\vdots \\
\nabla_{N_{g}} J_{N_{g}}\left(z_{N_{g}}, z_{-N_{g}}\right) \\
\nabla_{M O} J_{M O}\left(z_{M O}, z_{-M O}\right)
\end{array}\right]
$$


and $K=\prod_{i} K_{i}=\left(K_{1} \times K_{2} \times \cdots \times K_{N_{g}} \times K_{M O}\right)$, as defined before. The vector $F(z) \in \mathbb{R}^{2\left(N_{g}+1\right)}$ is also referred to as the "game map" for $\Gamma$. To prove the singleton nature of the solution set $\operatorname{SOL}(F, K)$ to the $\operatorname{VI}(F, K)$ (and by equivalence, as per [15, Proposition 1.4.2], uniqueness of Nash equilibria for $\Gamma$ ), we compute the Jacobian, $J F(z) \in \mathbb{R}^{2\left(N_{g}+1\right) \times 2\left(N_{g}+1\right)}$ of the game map, presented in Appendix A. As it can be observed, $J F(z)$ is symmetric which implies that there exists an equivalent optimization problem solving $\mathrm{VI}(K, F)$, whose first-order optimality conditions are given by $J F(z)$. This optimization problem is identical to $\mathcal{P}_{\text {cc }}$ which has a convex quadratic objective function and thus, has a unique minima. Thus, by equivalence of the optimization problem $\mathcal{P}_{\mathrm{cc}}$ with the $\operatorname{VI}(K, F)$ and game $\Gamma$ (per [15, Proposition 1.4.2]), we show that $\Gamma$ has a unique Nash equilibrium.

\section{B. Desirable Market Properties}

Building further on the discussion regarding existence and uniqueness of Nash equilibria, the following evaluates the satisfaction of the desirable market properties [16] by the solution to problem $\mathcal{P}_{\text {cc }}$.

Proposition 3 (Cost recovery for flexible generators): An optimal solution to $\mathcal{P}_{c c}$ ensures a non-negative payoff for the flexible generators under all possible market clearing outcomes and uncertainty realizations.

Proof: Mathematically, the non-negativity of payoff for flexible generators holds true if, at the optimal solution

$$
\begin{aligned}
& \left(\lambda^{\mathrm{DA} *} p_{g}^{*}+\lambda^{\mathrm{RP} *} \alpha_{g}^{*}\right) \\
& \quad-\left[C_{g}^{Q}\left(p_{g}^{* 2}+\left(\mathbf{e}^{\top} \Sigma \mathbf{e}\right) \alpha_{g}^{* 2}\right)+C_{g}^{L} p_{g}^{*}\right] \geq 0, \forall g,
\end{aligned}
$$

where the superscript $*$ indicates the optimal values for $p_{g}, \alpha_{g}, \lambda^{\mathrm{DA}}$ and $\lambda^{\mathrm{RP}}$. To prove the satisfaction of condition (9), we first formulate the dual problem for each flexible generator's profit maximization problem, discussed in (4). Defining a set of Lagrangian multipliers, $\Xi=$ $\left\{\underline{\mu_{g}}, \overline{\mu_{g}}, \rho_{g}^{\mathrm{DN}}, \rho_{g}^{\mathrm{UP}}, \chi_{g}, \overline{\chi_{g}}\right\}$ for the constraints (3d)-(3h), the dual problem can be formulated as

$$
\forall g \in \mathcal{G}\left\{\begin{array}{cl}
\min _{\Xi} . & \bar{\mu}_{g} p_{g}^{\max }+\rho_{g}^{\mathrm{DN}} R_{g}^{\mathrm{DN}, \max }+\rho_{g}^{\mathrm{UP}} R_{g}^{\mathrm{UP}, \max }+\overline{\chi_{g}} \\
\text { s.t. } & -\lambda^{\mathrm{DA}}+2 C_{g}^{Q} p_{g}+C_{g}^{L}-\underline{\mu}_{g}+\bar{\mu}_{g}=0: p_{g} \\
& -\lambda^{\mathrm{RP}}+2 C_{g}^{Q} \alpha_{g}-\underline{\chi}_{g}+\bar{\chi}_{g} \\
& +\Phi_{\left(1-\varepsilon_{g}\right)}^{-1} \sqrt{\mathbf{e}^{\top} \Sigma \mathbf{e}}\left(\underline{\mu}_{g}-\bar{\mu}_{g}\right. \\
& \left.-\rho_{g}^{\mathrm{DN}}+\rho_{g}^{\mathrm{UP}}\right)=0: \alpha_{g} \\
& \underline{\mu_{g}}, \overline{\mu_{g}}, \rho_{g}^{\mathrm{DN}}, \rho_{g}^{\mathrm{UP}}, \underline{\chi_{g}}, \overline{\chi_{g}} \geq 0 .
\end{array}\right.
$$

It should be noted that the dual problem (10) has an objective that is a sum of products of non-negative parameters and variables and thus, is always non-negative. Strong duality theory enforces primal (4) and dual (10) problems to have identical objective functions at optimal solution. Thus, (9) holds true for all market price and uncertainty outcomes.

Proposition 4 (Revenue adequacy for the MO): An optimal solution to $\mathcal{P}_{c c}$ ensures that the market operator never incurs a financial deficit for all possible market clearing outcomes and uncertainty realizations.
Proof: At the optimal solution to problem $\mathcal{P}_{\text {cc }}$, we multiply the equality constraints (3b) and (3c) with the optimal market clearing prices $\left(\lambda^{\mathrm{DA} *}, \lambda^{\mathrm{RP} *}\right)$ and subtract $(3 \mathrm{c})$ from ( $3 b)$ to obtain the following

$$
\begin{array}{r}
\left(\lambda^{\mathrm{DA} *} D-\lambda^{\mathrm{RP} *}\right)=\sum_{g \in \mathcal{G}} \lambda^{\mathrm{DA} *} p_{g}^{*} \\
+\sum_{k \in \mathcal{K}} \lambda^{\mathrm{DA} *} \hat{W}_{k} \\
-\sum_{g \in \mathcal{G}} \lambda^{\mathrm{RP} *} \alpha_{g}^{*} .
\end{array}
$$

The left-hand side of (11) is the total payment from loads less the reserve policy payments made to flexible generators by the market operator. Under any realization of wind forecast errors $\Delta$ in real-time, the right-hand side of (11) equals to the left-hand side considering that constraints (3b) and (3c) are strict equalities that are satisfied at the optimal solution. The MO, therefore, never incurs a financial deficit.

Proposition 5 (Market efficiency): Under the assumption of perfect competition, the market clearing problem $\mathcal{P}_{c c}$ ensures maximization of social welfare, such that no market participant desires to deviate from the market outcomes.

Proof: In an efficient market, social welfare is maximized and no market participant desires to deviate from the market outcomes, meaning that each market player maximizes their profit at the optimal solution of $\mathcal{P}_{\mathrm{cc}}$. This is proven, under the assumption of perfect competition among flexible generators, by the identical Karush-Kuhn-Tucker (KKT) optimality conditions of $\mathcal{P}_{\mathrm{cc}}$ and the equilibrium problem interpretation discussed in section II-D.1. The identical KKT conditions are presented in Appendix B.

Proposition 6 (Incentive compatibility): Under the assumption of perfect competition, the market clearing problem $\mathcal{P}_{c c}$ is such that each player can maximize its objective just by acting according to its "true" preferences.

Proof: Incentive compatibility implies that it is optimal for each participant to offer their production and reserves at a price equal to their "true" production cost. We define a bid made by a flexible generator $g$ to MO prior to market clearing $\mathcal{P}_{\mathrm{cc}}, B_{g}=\left[\left(C_{g}^{Q}, C_{g}^{L}\right), x_{g}\right]$ as a true-cost bid if the tuple $\left(C_{g}^{Q}, C_{g}^{L}\right)$ represents its true production cost. From the game interpretation of $\mathcal{P}_{\mathrm{cc}}$ discussed in section II-D.2, the cost function for generator $g$ at optimal solution $\left(x_{g}^{*}, \Lambda^{*}\right)$ is

$$
J_{g}\left(x_{g}^{*}, \Lambda^{*}\right)=\left(x_{g}^{* \top} Q_{g} x_{g}^{*}+L_{g}^{\top} x_{g}^{*}\right)-\Lambda^{* \top} x_{g}^{*}, \forall x_{g} \in K_{g} .
$$

If a generator were to bid, $\tilde{B}_{g}=\left[\left(\tilde{C}_{g}^{Q}, \tilde{C}_{g}^{L}\right), x_{g}\right]$ with a cost tuple $\left(\tilde{C}_{g}^{Q}, \tilde{C}_{g}^{L}\right)$ higher than its true-cost tuple, which is the only rational deviation from a true-cost bid, the resulting cost function at the solution is given by (13) below. Note that, under the assumption of perfect competition, the optimal market clearing prices $\Lambda^{*}$ are independent of a single generator's bid.

$$
J_{g}\left(\tilde{x}_{g}^{*}, \Lambda^{*}\right)=\left(\tilde{x}_{g}^{* \top} Q_{g} \tilde{x}_{g}^{*}+L_{g}^{\top} \tilde{x}_{g}^{*}\right)-\Lambda^{* \top} \tilde{x}_{g}^{*}, \forall x_{g} \in K_{g} .
$$

Comparing (13) with (12), we point out that $J_{g}\left(\tilde{x}_{g}^{*}, \Lambda^{*}\right) \geq$ $J_{g}\left(x_{g}^{*}, \Lambda^{*}\right)$ because from the proof of Proposition $1, \tilde{x}_{g}^{*}$ is a feasible suboptimal value for $x_{g}$. Thus, true-cost bidding is the dominant strategy for flexible generators. 


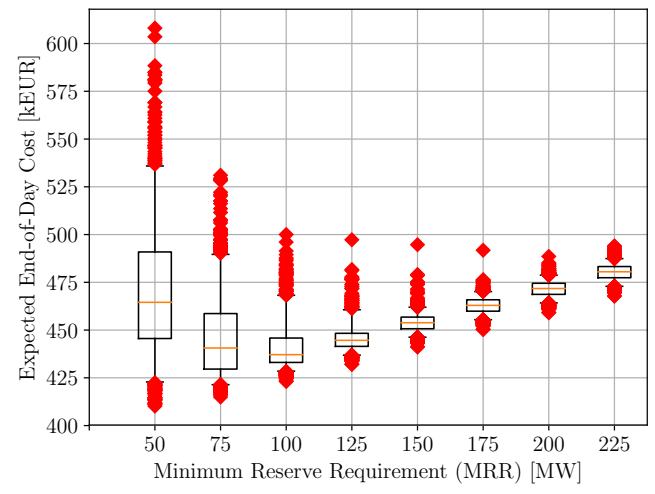

Fig. 3: Market clearing problem $\mathcal{P}_{\text {det }}$ : Average expected end-of-day operations cost and its variability, considering different values of MRR.

\section{CASE STUDY}

Data from a 24-bus system outlined in [23] is adapted to form a single-node electricity supply system with 6 wind farms. Each wind farm has an installed capacity of $200 \mathrm{MW}$ and the power production from it has a zero-mean normally distributed forecast error with standard deviation, $\sigma_{k}$ equal to $7.5 \%$ of installed capacity, as in [24]. Thus, problem $\mathcal{P}_{\text {cc }}$ is solved considering the standard deviation of total forecast error as $\Sigma=\sum_{k} \sigma_{k}$. The value of risk parameter, $\varepsilon_{g}$ for all generators is fixed as 0.05 . The peak demand in the simulation time horizon of 24 hours is $2,650 \mathrm{MW}$. The cost and capacity data of the flexible generators as well as the demand data is available as an online appendix in [25].

To highlight benefits of the proposed policy-based reserve procurement and operation, a deterministic benchmark $\left(\mathcal{P}_{\text {det }}\right)$ with energy and reserve co-optimization is set up. As formulated in Appendix C, the MO solves a day-ahead market clearing problem with an exogenous MRR, obtaining the optimal power dispatch as well as procuring the reserves needed. During real-time operation, through another marketbased mechanism, reserves are activated based on the bounds defined by day-ahead reserve allocation. In the absence of currently operational joint clearing of energy and reserves in the European electricity markets, this benchmark reflects a natural extension to the sequential market clearing approach.

The parameter MRR for the problem $\mathcal{P}_{\text {det }}$ is obtained by performing Out-of-Sample (OOS) simulations for 1000 scenarios of wind forecast errors to evaluate the expected end-of-day system operations cost while gradually increasing MRR, as shown in Figure 3. The orange line denotes the average value, boxes represent 25th and 75th percentiles and the whiskers extend to 5th and 95th percentiles. Red diamonds show the outliers. At low values of MRR, the expected cost of operation is observed to be high and has high variability due to load shedding in several scenarios of uncertainty realization, while at high values the cost increases due to the higher cost associated with over-dimensioning of reserve requirements. For comparison with results from the market clearing proposed in this paper, we fix the value of MRR at $200 \mathrm{MW}$.

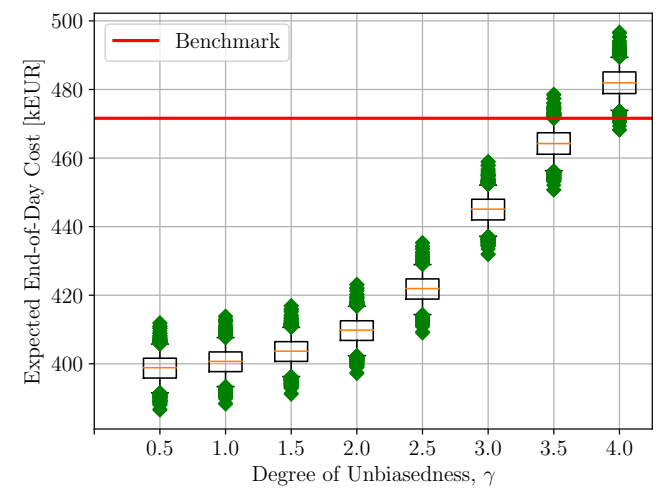

Fig. 4: Market clearing problem $\mathcal{P}_{\text {cc }}$ : Average expected end-of-day operations cost and its variability, considering different values of $\gamma$.

First, biased OOS simulations ${ }^{2}$ are performed wherein the 1000 scenarios for wind forecast errors are drawn from a Normal distribution identical to that assumed by the chance constraints in problem $\mathcal{P}_{\mathrm{cc}}$ for reformulation of the objective (3a) and chance constraints (3d)-(3g). It is expected that market clearing problem $\mathcal{P}_{\text {cc }}$ should outperform $\mathcal{P}_{\text {det }}$, resulting in lower system operations cost. This stems from the fact that problem $\mathcal{P}_{\text {cc }}$ provides an optimal solution with respect to this uncertainty distribution.

To study unbiased OOS simulations, considering the same set of scenarios, we replace the standard deviation, $\Sigma$ in problem $\mathcal{P}_{\mathrm{cc}}$ with $(\gamma * \Sigma)$, such the parameter $\gamma$ represents the degree of unbiasedness of the probability distribution assumed in $\mathcal{P}_{\text {cc }}$. Figure 4 shows the variation in expected endof-day system operations cost with $\gamma$ (green diamonds show the outliers). The red horizontal line indicates the average expected end-of-day cost for the benchmark market clearing $\mathcal{P}_{\text {det }}$, operating with an MRR, $M_{R}=200 \mathrm{MW}$. The case of $\gamma=1$ is equivalent to the previously discussed biased OOS, considering that the probability distribution assumed in problem $\mathcal{P}_{\text {cc }}$ is identical to actual wind forecast errors. For $\gamma<1$, the problem $\mathcal{P}_{\text {cc }}$ underestimates the actual wind forecast errors, resulting in lower costs of day-ahead reserve procurement. However, with the reserve control policy in place, the MO is able to successfully mitigate the imbalances in real-time at a low cost. For values of $\gamma$ larger than 1, the problem $\mathcal{P}_{\mathrm{cc}}$ overestimates the actual wind forecast errors, thus resulting in higher costs of reserve procurement to ensure mitigation of imbalances in real-time. For $\gamma>4$, we observed infeasibility of the market clearing problem $\mathcal{P}_{c c}$ as we reach the limits of available reserve capacity from the generators to successfully mitigate the imbalance. Table I presents a comparison of average expected system operations cost for market clearing using problem $\mathcal{P}_{\text {det }}$ with that using $\mathcal{P}_{c c}$ for a selection of values of $\gamma$. As it can be observed, market clearing problem $\mathcal{P}_{\text {cc }}$ leads to a reduction of $5.4 \%$ in the average expected end-of-day system operations cost

\footnotetext{
${ }^{2}$ Here, the notion of OOS simulations refers to the different second moments, $\Sigma$ of the Normal distribution from which scenarios of actual wind forecast errors are drawn. In contrast, stochastic programming literature typically considers random scenarios drawn from other distributions as OOS.
} 
TABLE I: Comparison between average expected costs obtained from $\mathcal{P}_{\text {det }}$ and $\mathcal{P}_{\mathrm{cc}}$

\begin{tabular}{|l|c|c|c|c|}
\hline Costs $[\mathrm{k} €]$ & $\begin{array}{c}\mathcal{P}_{\text {det }} \\
M_{R}=200 \mathrm{MW}\end{array}$ & $\begin{array}{c}\mathcal{P}_{\mathrm{cc}} \\
\gamma=0.5\end{array}$ & $\begin{array}{c}\mathcal{P}_{\mathrm{cc}} \\
\gamma=1.0\end{array}$ & $\begin{array}{c}\mathcal{P}_{\mathrm{cc}} \\
\gamma=3.0\end{array}$ \\
\hline Operations & 401.4 & 398.2 & 400.9 & 408.0 \\
\hline Reserves & 70.2 & 0.6 & 2 & 38.1 \\
\hline Total & 471.6 & 398.6 & 402.9 & 446.1 \\
\hline Change $[\%]$ & - & $\mathbf{- 1 5 . 5 \%}$ & $\mathbf{- 1 4 . 6 \%}$ & $\mathbf{- 5 . 4 \%}$ \\
\hline
\end{tabular}
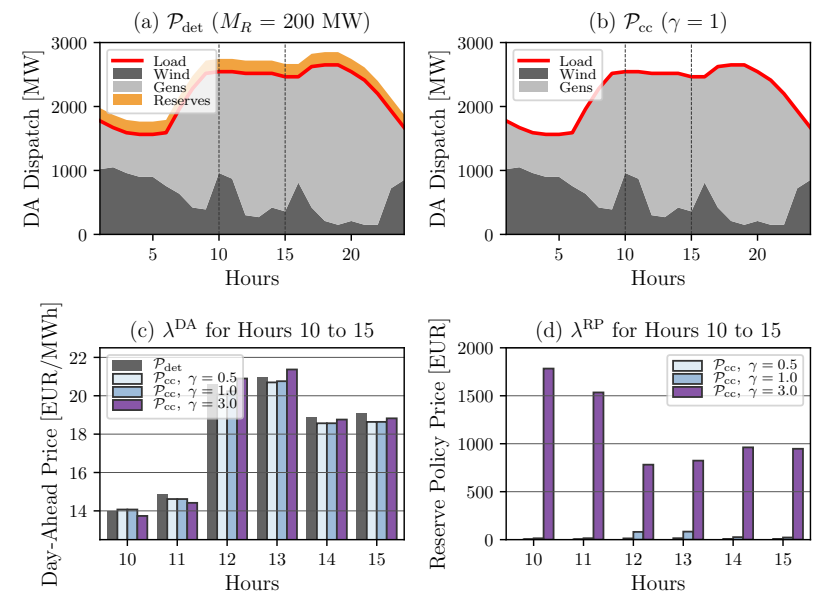

Fig. 5: $\mathcal{P}_{\text {det }}, \mathcal{P}_{\text {cc }}$ : Day-ahead (DA) dispatch and market prices

compared to $\mathcal{P}_{\text {det }}$, even if the actual wind forecast errors are over-estimated by a factor of $3(\gamma=3)$. Results of the case study demonstrate the resilience of proposed market clearing problem $\mathcal{P}_{\text {cc }}$ to large forecast errors within the normally distributed forecast error assumption.

A short discussion on the day-ahead market clearing outcomes of $\mathcal{P}_{\text {det }}$ and $\mathcal{P}_{\text {cc }}$ follows. Figures $5 \mathrm{a}$ and $5 \mathrm{~b}$ show a comparison of the optimal dispatch of the flexible generators and wind farms to meet the inflexible load for $\mathcal{P}_{\text {det }}$ and $\mathcal{P}_{\text {cc }}$. While reserve capacity is scheduled in the $\mathcal{P}_{\text {det }}$ case, in $\mathcal{P}_{\text {cc }}$ explicit capacity reserve procurement is replaced by control policies, characterized by $\alpha_{g}$. Optimal day-ahead price for energy, $\lambda^{\mathrm{DA}}$ and for the reserve policies, $\lambda^{\mathrm{RP}}\left(\right.$ in $\mathcal{P}_{\text {cc }}$ ) for Hours 10 through 15 are shown in Figures $5 \mathrm{c}$ and 5d. Hours with higher wind power forecast, $\sum_{k \in \mathcal{K}} \hat{W}_{k}$, result in lower prices, $\lambda^{\mathrm{DA}}$ for both $P_{\mathrm{det}}$ and $P_{\mathrm{cc}}$, owing to the zero marginal cost of wind farms. Moreover, as observed in Figure 5d, higher values of the $\gamma$ result in a higher price of reserve policies, $\lambda^{\mathrm{RP}}$ signifying the overestimation of actual wind forecast errors by the market clearing problem, $\mathcal{P}_{\text {cc. }}$. Figure 6 shows the allocation of the participation factors, $\alpha_{g}$ for the same hours among the generators having available reserve capacity. It is observed that the more expensive generator G4 is allocated non-zero $\alpha_{g}$ in the hours with high share of wind and with higher values of $\gamma$.

\section{CONCLUSION}

We proposed a market clearing mechanism for day-ahead electricity markets that co-optimizes energy and control policy-based reserves using chance-constrained optimization techniques. Using tools from equilibrium modeling and VIs, we proved the existence and uniqueness of Nash equilibria for this market clearing. No desirable properties inherent

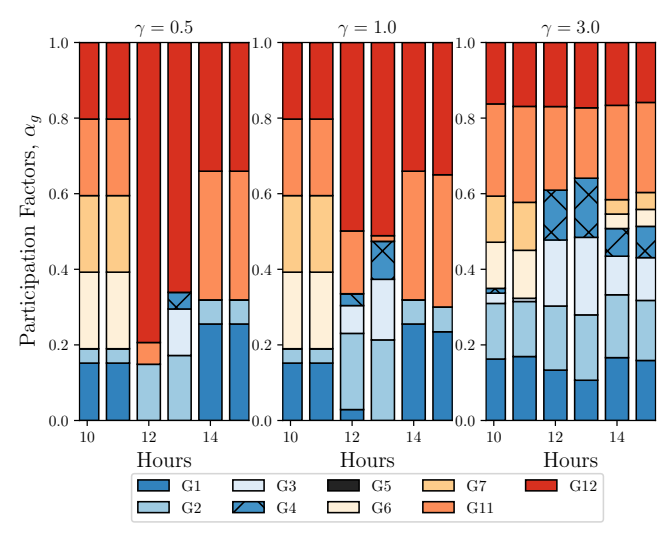

Fig. 6: Allocation of participation factors, $\alpha_{g}$ with $\gamma$

to the prevalent deterministic market clearing are lost in the proposed chance-constrained policy-based market clearing. Under assumptions of proper uncertainty modeling, we demonstrated the reduction in average expected system operations cost over a deterministic benchmark.

In future, the suggested non-requirement of energy redispatch should be further studied to determine its impact on system reliability and primary frequency control, considering that the estimation of the true moments of uncertainty is challenging. Evaluation of the properties of proposed policy-based reserve procurement considering the temporal and spatial correlation of uncertainty, a power system with congested line capacity limits and generic control policies defined to include other flexibility sources (e.g. demand response, energy storage) also remain topics for future work.

\section{APPENDIX}

\section{A. Game Map for $\Gamma$}

The Jacobian matrix, $J F(z)$, of the game map $F(z)$ for the non-cooperative game $\Gamma$, discussed in section III-A is

$$
\left[\begin{array}{ccccccc}
2 C_{1}^{Q} & 0 & \cdots & 0 & 0 & -1 & 0 \\
0 & 2 C_{1}^{Q}\left(\mathbf{e}^{\top} \Sigma \mathbf{e}\right) & \cdots & 0 & 0 & 0 & -1 \\
\vdots & \ddots & \ddots & \ddots & \ddots & \vdots & \vdots \\
0 & 0 & \cdots & 2 C_{N_{g}}^{Q} & 0 & -1 & 0 \\
0 & 0 & \cdots & 0 & 2 C_{N_{g}}^{Q}\left(\mathbf{e}^{\top} \Sigma \mathbf{e}\right) & 0 & -1 \\
-1 & 0 & \cdots & -1 & 0 & 0 & 0 \\
0 & -1 & \cdots & 0 & -1 & 0 & 0
\end{array}\right]
$$

\section{B. KKTs of Optimization Problem $\mathcal{P}_{c c}$}

Considering $\mathcal{L}$ as the Lagrangian function for the optimization problem $\mathcal{P}_{\mathrm{cc}}$, its KKT optimality conditions are given by (14). Its equivalent equilibrium problem (discussed in section II-D.1) has an identical set of KKT conditions.

$$
\begin{aligned}
& (3 \mathrm{~b})-(3 \mathrm{c}) \\
& \frac{\partial \mathcal{L}}{\partial p_{g}}=2 C_{g}^{Q} p_{g}+C_{g}^{L}-\lambda^{\mathrm{DA}}-\underline{\mu}_{g}+\bar{\mu}_{g}=0, \forall g \\
& \frac{\partial \mathcal{L}}{\partial \alpha_{g}}=2 C_{g}^{Q} \alpha_{g}-\lambda^{\mathrm{RP}}-\underline{\chi}_{g}+\bar{\chi}_{g}+\Phi_{\left(1-\varepsilon_{g}\right)}^{-1} \sqrt{\mathbf{e}^{\top} \Sigma \mathbf{e}}\left(\underline{\mu}_{g}\right. \\
& \left.\quad-\bar{\mu}_{g}-\rho_{g}^{\mathrm{DN}}+\rho_{g}^{\mathrm{UP}}\right)=0, \forall g
\end{aligned}
$$




$$
\begin{aligned}
& 0 \leq\left(p_{g}-\Phi_{\left(1-\varepsilon_{g}\right)}^{-1} \alpha_{g} \sqrt{\left(\mathbf{e}^{\top} \Sigma \mathbf{e}\right)}\right) \perp \underline{\mu}_{g} \geq 0, \forall g \\
& 0 \leq\left(p_{g}^{\max }-p_{g}-\Phi_{\left(1-\varepsilon_{g}\right)}^{-1} \alpha_{g} \sqrt{\left(\mathbf{e}^{\top} \Sigma \mathbf{e}\right)}\right) \perp \bar{\mu}_{g} \geq 0, \forall g \\
& 0 \leq\left(R_{g}^{\mathrm{DN}, \max }+\Phi_{\left(1-\varepsilon_{g}\right)}^{-1} \alpha_{g} \sqrt{\left(\mathbf{e}^{\top} \Sigma \mathbf{e}\right)}\right) \perp \rho_{g}^{\mathrm{DN}} \geq 0, \forall g \\
& 0 \leq\left(R_{g}^{\mathrm{UP}, \max }-\Phi_{\left(1-\varepsilon_{g}\right)}^{-1} \alpha_{g} \sqrt{\left(\mathbf{e}^{\top} \Sigma \mathbf{e}\right)}\right) \perp \rho_{g}^{\mathrm{UP}} \geq 0, \forall g \\
& 0 \leq \alpha_{g} \perp \underline{\chi}_{g} \geq 0, \forall g \\
& 0 \leq\left(1-\alpha_{g}\right) \perp \bar{\chi}_{g} \geq 0, \forall g .
\end{aligned}
$$

\section{Deterministic Benchmark, $\mathcal{P}_{\text {det }}$}

The day-ahead deterministic co-optimization of energy and reserves solved by the MO is given in problem (15).

$$
\begin{array}{ll}
\min _{p_{g}, R_{g}} & \sum_{g \in \mathcal{G}}\left[C_{g}^{Q} p_{g}^{2}+C_{g}^{L} p_{g}+C_{g}^{R} R_{g}\right] \\
\text { s.t. } & \sum_{g \in \mathcal{G}} p_{g}+\sum_{k \in \mathcal{K}} \hat{W}_{k}=D, \quad: \lambda^{\mathrm{DA}} \\
& \sum_{g \in \mathcal{G}} R_{g} \geq M_{R} \\
& 0 \leq p_{g} \leq p_{g}^{\max }, \forall g \in \mathcal{G} \\
& 0 \leq R_{g} \leq R_{g}^{\max }, \forall g \in \mathcal{G} \\
& p_{g}+R_{g} \leq p_{g}^{\max }, \forall g \in \mathcal{G} \\
& p_{g}-R_{g} \geq 0, \forall g \in \mathcal{G},
\end{array}
$$

where the MRR, $M_{R}$ is an exogenous parameter, $C_{g}^{R}$ denotes the cost of reserve procurement and $\left(p_{g}, R_{g}\right)$ represents the set of power dispatch and reserve allocation for generator $g$. The real-time balancing market clearing, formulated in problem (16), activates the reserve energy from flexible generators, $r_{g}$, limited to the optimal reserve capacities $R_{g}^{*}$ allocated day ahead (constraint (16c)). Further, considering that the actual forecast error $\Delta$ could take extreme values in some cases, real-time balancing allows for wind spillage, $W_{k}^{\mathrm{sp}}$ at zero cost and load shedding, $D^{\text {sh }}$ at $C^{\mathrm{VoLL}}=€ 500 / \mathrm{MWh}$.

$$
\begin{array}{ll}
\min _{r_{g}, D^{\mathrm{sh}}, W_{k}^{\mathrm{sp}}} & \sum_{g \in \mathcal{G}}\left[C_{g}^{Q}\left(P_{g}^{*}+r_{g}\right)^{2}+C_{g}^{L}\left(P_{g}^{*}+r_{g}\right)\right]+C^{\mathrm{VoLL}} D^{\mathrm{sh}} \\
& \sum_{g \in \mathcal{G}} r_{g}=\Delta \\
& -R_{g}^{*} \leq r_{g} \leq R_{g}^{*}, \forall g \in \mathcal{G} \\
& 0 \leq D^{\mathrm{sh}} \leq D \\
& 0 \leq W_{k}^{\mathrm{sp}} \leq\left(\hat{W}_{k}+\delta_{k}\right), \forall k \in \mathcal{K} .
\end{array}
$$

\section{REFERENCES}

[1] P. González, J. Villar, C. A. Díaz, and F. A. Campos, "Joint energy and reserve markets: Current implementations and modeling trends," Electric Power Systems Research, vol. 109, pp. 101-111, Apr. 2014.

[2] J. Morales, M. Zugno, S. Pineda, and P. Pinson, "Electricity market clearing with improved scheduling of stochastic production," European Journal of Operational Research, vol. 235, pp. 765-774, Jun. 2014.

[3] A. Papavasiliou, S. S. Oren, and R. P. O'Neill, "Reserve requirements for wind power integration: A scenario-based stochastic programming framework," IEEE Transactions on Power Systems, vol. 26, pp. 21972206, Nov. 2011.
[4] R. Domínguez, G. Oggioni, and Y. Smeers, "Reserve procurement and flexibility services in power systems with high renewable capacity: Effects of integration on different market designs," International Journal of Electrical Power \& Energy Systems, vol. 113, pp. 10141034, 2019.

[5] A. Virag, A. Jokić, R. M. Hermans, and P. P. J. van den Bosch, "Combined bidding at power and ancillary service markets," in 8th International Conference on the European Energy Market (EEM), pp. 568-573, May 2011.

[6] J. M. Morales, A. J. Conejo, and J. Perez-Ruiz, "Economic valuation of reserves in power systems with high penetration of wind power," IEEE Transactions on Power Systems, vol. 24, pp. 900-910, May 2009.

[7] F. Abbaspourtorbati and M. Zima, "The Swiss reserve market: Stochastic programming in practice," IEEE Transactions on Power Systems, vol. 31, pp. 1188-1194, Mar. 2016.

[8] J. Warrington, P. Goulart, S. Marithoz, and M. Morari, "Policy-based reserves for power systems," IEEE Transactions on Power Systems, vol. 28, pp. 4427-4437, Nov. 2013.

[9] J. Warrington, C. Hohl, P. J. Goulart, and M. Morari, "Optimal unit commitment accounting for robust affine reserve policies," in 2014 American Control Conference, pp. 5049-5055, Jun. 2014.

[10] D. Bertsimas, E. Litvinov, X. A. Sun, J. Zhao, and T. Zheng, "Adaptive robust optimization for the security constrained unit commitment problem," IEEE Transactions on Power Systems, vol. 28, pp. 52-63, Feb. 2013.

[11] D. Bienstock, M. Chertkov, and S. Harnett, "Chance-constrained optimal power flow: Risk-aware network control under uncertainty," SIAM Review, vol. 56, no. 3, pp. 461-495, 2014.

[12] K. Margellos, P. Goulart, and J. Lygeros, "On the road between robust optimization and the scenario approach for chance constrained optimization problems," IEEE Transactions on Automatic Control, vol. 59, pp. 2258-2263, Aug. 2014

[13] A. Nemirovski, "On safe tractable approximations of chance constraints," European Journal of Operational Research, vol. 219, no. 3, pp. $707-718,2012$. Feature Clusters.

[14] A. Georghiou, D. Kuhn, and W. Wiesemann, "The decision rule approach to optimization under uncertainty: Methodology and applications," Computational Management Science, Nov. 2018.

[15] F. Facchinei and J.-S. Pang, Finite-Dimensional Variational Inequalities and Complementarity Problems. New York, NY: Springer New York, 2003.

[16] F. C. Schweppe, M. C. Caramanis, R. D. Tabors, and R. E. Bohn, Spot Pricing of Electricity. Boston, MA: Springer US, 1988.

[17] X. Kuang, Y. Dvorkin, A. J. Lamadrid, M. A. Ortega-Vazquez, and L. F. Zuluaga, "Pricing chance constraints in electricity markets," IEEE Transactions on Power Systems, vol. 33, pp. 4634-4636, Jul. 2018.

[18] M. Lubin, Y. Dvorkin, and S. Backhaus, "A robust approach to chance constrained optimal power flow with renewable generation," IEEE Transactions on Power Systems, vol. 31, pp. 3840-3849, Sep. 2016.

[19] A. Prékopa, Convexity Theory of Probabilistic Constrained Problems, vol. 324, pp. 301-317. Dordrecht, The Netherlands: Springer, 1995.

[20] B. F. Hobbs, "Linear complementarity models of Nash-Cournot competition in bilateral and POOLCO power markets," IEEE Transactions on Power Systems, vol. 16, pp. 194-202, May 2001.

[21] J. Kazempour, P. Pinson, and B. F. Hobbs, "A stochastic market design with revenue adequacy and cost recovery by scenario: Benefits and costs," IEEE Transactions on Power Systems, vol. 33, pp. 3531-3545, Jul. 2018.

[22] J. B. Rosen, "Existence and uniqueness of equilibrium points for concave N-person games," Econometrica, vol. 33, pp. 520-534, 1965.

[23] C. Ordoudis, P. Pinson, J. Morales González, and M. Zugno, An Updated Version of the IEEE RTS 24-Bus System for Electricity Market and Power System Operation Studies. Technical University of Denmark (DTU), 2016.

[24] U. Focken, M. Lange, K. Monnich, H. Waldl, H. Beyer, and A. Luig, "Short-term prediction of the aggregated power output of wind farms - A statistical analysis of the reduction of the prediction error by spatial smoothing effects," Journal of Wind Engineering and Industrial Aerodynamics, vol. 90, pp. 231-246, Mar. 2002.

[25] A. Ratha, J. Kazempour, A. Virag, and P. Pinson, "Online appendix for paper: Exploring market properties of policy-based reserve procurement for power systems," 2019. [Online]. Available: https: //doi.org/10.5281/zenodo.2595186. 